\title{
Transition to ultimate Rayleigh-Bénard turbulence revealed through extended self-similarity scaling analysis of the temperature structure functions
}

\author{
Dominik Krug ${ }^{1,2,} \dagger$, Xiaojue Zhu ${ }^{1}$, Daniel Chung ${ }^{2}$, Ivan Marusic ${ }^{2}$, \\ Roberto Verzicco $^{1,3}$ and Detlef Lohse ${ }^{1,4}$ \\ ${ }^{1}$ Physics of Fluids Group and Twente Max Planck Center, Department of Science and Technology, \\ Mesa+ Institute, and J.M. Burgers Center for Fluid Dynamics, University of Twente, PO Box 217, \\ 7500 AE Enschede, The Netherlands \\ ${ }^{2}$ Department of Mechanical Engineering, University of Melbourne, 3010 Victoria, Australia \\ ${ }^{3}$ Dipartimento di Ingegneria Industriale, University of Rome 'Tor Vergata', Via del Politecnico 1 \\ Roma 00133, Italy \\ ${ }^{4}$ Max Planck Institute for Dynamics and Self-Organization, 37077 Göttingen, Germany
}

(Received 28 April 2018; revised 9 July 2018; accepted 10 July 2018;

first published online 30 July 2018)

In turbulent Rayleigh-Bénard (RB) convection, a transition to the so-called ultimate regime, in which the boundary layers $(\mathrm{BL})$ are of turbulent type, has been postulated. Indeed, at very large Rayleigh number $R a \approx 10^{13}-10^{14}$ a transition in the scaling of the global Nusselt number $N u$ (the dimensionless heat transfer) and the Reynolds number with $R a$ has been observed in experiments and very recently in direct numerical simulations (DNS) of two-dimensional (2D) RB convection. In this paper, we analyse the local scaling properties of the lateral temperature structure functions in the BLs of this simulation of 2D RB convection, employing extended self-similarity (ESS) (i.e., plotting the structure functions against each other, rather than only against the scale) in the spirit of the attached-eddy hypothesis, as we have recently introduced for velocity structure functions in wall turbulence (Krug et al., J. Fluid Mech., vol. 830, 2017, pp. 797-819). We find no ESS scaling at $R a$ below the transition and in the near-wall region. However, beyond the transition and for large enough wall distance $z^{+}>100$, we find clear ESS behaviour, as expected for a scalar in a turbulent boundary layer. In striking correspondence to the $N u$ scaling, the ESS scaling region is negligible at $R a=10^{11}$ and well developed at $R a=10^{14}$, thus providing strong evidence that the observed transition in the global Nusselt number at $R a \approx 10^{13}$ indeed is the transition from a laminar type BL to a turbulent type BL. Our results further show that the relative slopes for scalar structure functions in the ESS scaling regime

$†$ Email address for correspondence: d.j.krug@utwente.nl 


\section{Krug, X. Zhu, D. Chung, I. Marusic, R. Verzicco and D. Lohse}

are the same as for their velocity counterparts, extending their previously established universality. The findings are confirmed by comparing to scalar structure functions in three-dimensional turbulent channel flow.

Key words: Bénard convection, turbulent boundary layers, turbulent flows

\section{Introduction}

Thermal convection is relevant to a wide range of applications across various fields, such as building ventilation (e.g. Linden 1999), atmospheric (e.g. Hartmann, Moy \& Fu 2001) or oceanic (e.g. Rahmstorf 2000) flows. The phenomenon is widely studied in terms of the paradigmatic case of Rayleigh-Bénard (RB) convection (see the reviews of Ahlers, Grossmann \& Lohse 2009; Lohse \& Xia 2010; Chillà \& Schumacher 2012), in which two horizontal plates at a distance $L$ are cooled from above and heated from below. In the presence of a gravitational acceleration $g$, a flow is driven with properties (for given Prandtl number $\operatorname{Pr} \equiv \nu / \kappa$ and aspect ratio $\Gamma=D / L)$ that are controlled by the Rayleigh number $R a \equiv \alpha g \Delta L^{3} \Theta /(\nu \kappa)$. Here, $\alpha$ is the thermal expansion coefficient, $v$ and $\kappa$ are kinematic viscosity and the thermal expansion coefficient, respectively, $D$ is some lateral length scale and $\Delta \Theta \equiv \Theta_{b}-\Theta_{t}$ denotes the temperature difference between the bottom (held at $\Theta_{b}$ ) and top $\left(\Theta_{t}\right)$ plates. The primary response parameter of the system is the resulting heat flux, which in its non-dimensional form is given by the Nusselt number $N u$ relating the actual heat transfer to the one in the purely conductive case. The behaviour of the system at very high $R a$ is of interest in many applications, and theoretical work (Kraichnan 1962; Spiegel 1971; Grossmann \& Lohse 2000, 2011) predicts the existence of a so-called 'ultimate regime', in which the scaling $N u \propto R a^{\beta}$ switches from the classical $\beta \leqslant 1 / 3$ (Malkus 1954) to $\beta>1 / 3$. This transition is related to a change of the boundary layer (BL) structure, of both velocity and temperature, from a laminar to turbulent type. A transition in the $N u$ scaling - as well as in the Reynolds number scaling - has indeed been observed experimentally, most convincingly by He et al. (2012a,b, 2015) and very recently also in a direct numerical simulation (DNS) of two-dimensional (2D) RB convection by Zhu et al. (2018). Results of the latter are reproduced in figure $1(a)$, where the transition is evident from the change in slope of the (compensated) $N u$ at $R a \approx 10^{13}$. As expected from the theory and indicative of the turbulent nature of the BLs, Zhu et al. (2018) found logarithmic dependencies with respect to the distance from the wall for the mean temperature and velocity BLs. In the following, we will characterize the thermal BLs further by studying their lateral structure functions.

Such an analysis originated from Davidson, Nickels \& Krogstad (2006), who pointed out that the $k^{-1}$ spectral scaling ( $k$ being the streamwise wavenumber) of the streamwise velocity power spectrum predicted by the attached-eddy hypothesis (Townsend 1976; Perry \& Chong 1982), is equivalent to - and more readily observed as $-\mathrm{a} \ln (r / z)$ scaling of the second-order longitudinal structure function. Here and in the following, $r$ denotes the streamwise separation distance and $z$ is the distance off the wall. De Silva et al. (2015) found that the $\ln (r / z)$ scaling also applies to velocity structure functions of arbitrary even order $2 p$ such that for the so-called energy-containing range $z<r \ll \delta$ ( $\delta$ being the boundary layer thickness, estimated to be of order of the gap half-width $L / 2$ here)

$$
S_{p}^{u} \equiv\left\langle\Delta u^{2 p}\right\rangle^{1 / p} / U_{\tau}^{2}=E_{p}^{u}+D_{p}^{u} \ln \frac{r}{z} .
$$



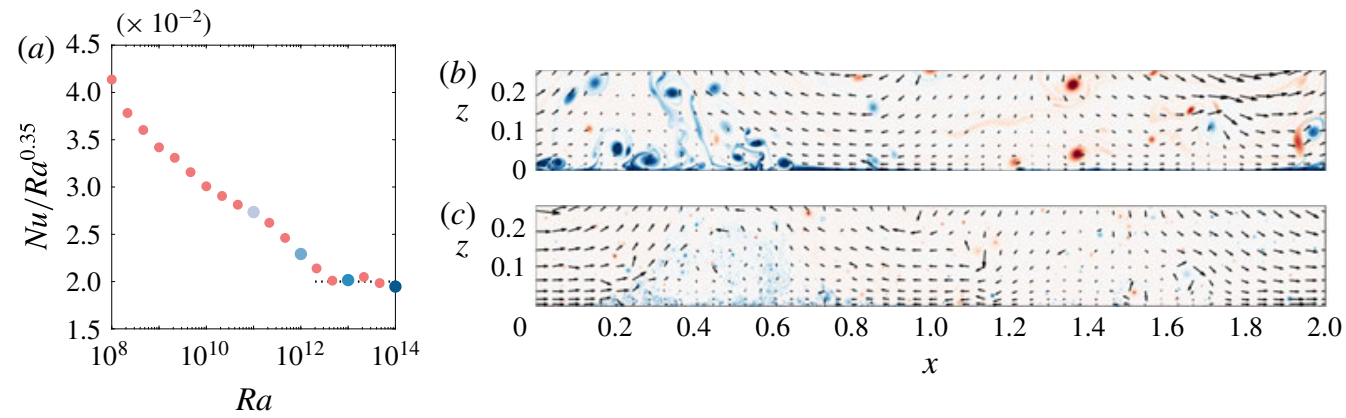

FIgURE 1. (a) Reduced Nusselt number $N u / R a^{0.35}$ from Zhu et al. (2018); blue symbols indicate the $R a$ values investigated here. Note that the compensation with $R a^{0.35}$ is chosen to highlight the transition and does not imply that the $N u$ versus $R a$ scaling saturates at this exponent. $(b, c)$ Instantaneous snapshots of temperature (contours for $0.3 \leqslant \theta / \Delta \Theta \leqslant 0.7$ from blue to red) with rescaled velocity vectors at $R a=10^{11}(b)$ and $R a=10^{14}(c)$. Only a quarter of the vertical domain (z-direction) is shown.

Here $\Delta u(r, z)$ is the velocity increment between two points at a distance $z$ off the wall separated by $r$ along the streamwise direction, $U_{\tau}$ is the mean friction velocity and $E_{p}^{u}, D_{p}^{u}$ are constants. We use superscript $u$ to denote quantities relating to the velocity and $\theta$ when referring to the scalar later on. Note that (1.1) applies for scales larger than the inertial subrange $\eta \ll r \ll z$, with $\eta$ being the Kolmogorov microscale (for an overview of all scaling regimes see Davidson et al. (2006)).

While the direct scaling according to (1.1) is only observed at relatively large Reynolds numbers $R e_{\tau}=\delta U_{\tau} / v \sim O\left(10^{4}\right)$, Krug et al. (2017) and de Silva et al. (2017) have shown that a relative scaling is evident at much smaller $\operatorname{Re}_{\tau}$ if the so-called extended self-similarity (ESS) framework is employed. In this case, the scaling is not analysed as a function of $r$ but - in the spirit of the ESS hypothesis originally conceived for the Kolmogorov inertial range by Benzi et al. (1993, 1995) - relative to a structure function of different order. For an arbitrary reference order $2 m$ this results in the 'ESS form' of (1.1), namely

$$
S_{p}^{u}=\frac{D_{p}^{u}}{D_{m}^{u}} S_{m}^{u}+E_{p}^{u}-\frac{D_{p}^{u}}{D_{m}^{u}} E_{m}^{u} .
$$

Krug et al. (2017) and de Silva et al. (2017) found that the linear scaling of (1.2) could not only be observed at relatively low $R e_{\tau}$ well within the capabilities of current DNS, but also that the relative slopes $D_{p}^{u} / D_{m}^{u}$ exhibit non-trivial, i.e. non-Gaussian, universal behaviour across various flow geometries, such as flat-plate boundary layers, pipe and channel flow and even Taylor-Couette flow. Krug et al. (2017) identified the fact that (1.2) relaxes a self-similarity assumption required for (1.1) as a potential explanation for the efficacy of the ESS formulation.

From our experience in Taylor-Couette flow (Krug et al. 2017) we learnt that ESS scaling according to (1.2) is not observed if large-scale structures in the bulk (such as the Taylor rolls) contribute to the velocity component under investigation. This is also the case for the wall-parallel velocity component in RB convection, as can be seen from the snapshots in figure $1(b, c)$. Since no equivalent scaling exists for the wall-normal component (Perry \& Chong 1982), the 2D velocity field in RB convection 


\section{Krug, X. Zhu, D. Chung, I. Marusic, R. Verzicco and D. Lohse}

is ruled out as a suitable candidate for this scaling analysis. Therefore, and due to the fact that velocity structure functions are found to be significantly less converged, the current study instead focuses on structure functions of the temperature field

$$
S_{p}^{\theta}(r) \equiv\left\langle\Delta \theta^{2 p}\right\rangle^{1 / p} / \Theta_{\tau}^{2}
$$

Here, $\Delta \theta$ and $\Theta_{\tau}=-\left.\kappa \partial_{z} \Theta\right|_{z=0} / U_{\tau}$ are analogous to $\Delta u$ and $U_{\tau}$, respectively. ESS scaling of scalar structure functions in the energy-containing range has not been considered yet. It is, however, conceivable that the situation will be similar to velocity structure functions: the theory underlying (1.1) and (1.2) is based on an inertial assumption, which implies that momentum transport is dominated by turbulent eddies that are larger than and therefore constrained by $z \gg \eta$ but smaller than $\delta$. In the spirit of the Reynolds analogy and for $\operatorname{Pr} \approx 1$ these eddies can be assumed to affect momentum and scalar similarly. Consequently, the relevant arguments based on the attached-eddy hypothesis (see de Silva et al. 2015; Krug et al. 2017) can be expected to transfer to the scalar field as well. It is therefore our objective here to investigate whether and at what $R a$ the thermal boundary layers in RB convection exhibit an ESS scaling according to

$$
S_{p}^{\theta}=\frac{D_{p}^{\theta}}{D_{m}^{\theta}} S_{m}^{\theta}+E_{p}^{\theta}-\frac{D_{p}^{\theta}}{D_{m}^{\theta}} E_{m}^{\theta} .
$$

The expectation is that such a scaling regime should originate coinciding with the $\mathrm{Nu}$ scaling transition, which has been link to the emergence of logarithmic boundary layers (Grossmann \& Lohse 2011). Before moving ahead, we would like to mention that the attached-eddy framework, from which (1.1) and (1.2) can be derived (see Krug et al. 2017), is not new in the RB context. It has already been referred to by Ahlers, Bodenschatz \& He (2014), who investigate logarithmic dependencies of temperature profiles, and by $\mathrm{He}$ et al. (2014) in a study of $f^{-1}$ temperature power spectra scalings (the temporal equivalent to the $k^{-1}$ scaling) in order to interpret the observations made.

Since the dataset of Zhu et al. (2018) that will be employed for this endeavour is $2 \mathrm{D}$, we will also check our results by using a three-dimensional (3D) channel simulation as a reference and for comparison. A brief overview over both datasets will given in $\S 2$, before presenting our results in $\S 3$ and conclusions in $\S 4$.

\section{Datasets}

The simulations of Zhu et al. (2018) were performed using the second-order finite-difference code AFiD (van der Poel et al. 2015) on a 2D domain with periodic boundary conditions in the lateral direction with $\Gamma=2$ and $\operatorname{Pr}=1$.

While the original dataset spans six decades $R a \in\left[10^{8}, 10^{14}\right]$, only four data points for $R a \geqslant 10^{11}$ are used here (see figure $1 a$ ). We refer to the original publication for further details on the numerical set-up and validation of the results. One of the particularities of the 2D set-up is that the large-scale structures, which can be observed in the vector maps of figure $1(b, c)$, remain almost fixed in place, allowing for simple temporal averaging. Since the temporal mean velocity gradient changes sign along the plate, we take the spatial mean of its absolute value when computing $U_{\tau}$ in RB convection. For the present simulations we obtain friction Reynolds numbers $R e_{\tau} \equiv L^{+} / 2=L U_{\tau} /(2 v) \approx[2400 ; 5700 ; 12300 ; 34400]$ at $R a=\left[10^{11} ; 10^{12} ; 10^{13} ; 10^{14}\right]$, respectively. 


\section{ESS scaling of temperature structure functions in RB turbulence}

Differences and similarities between 2D and 3D RB convection are discussed in detail by Schmalzl, Breuer \& Hansen (2004) and van der Poel, Stevens \& Lohse (2013). Here we only note that, with the exception of flows at high $\operatorname{Pr}, 2 \mathrm{D}$ simulations of RB flow capture the integral behaviour well. In view of the fact that the PrandtlBlasius boundary layer theory (Schlichting \& Gersten 2000) as well as the additional scaling arguments put forward in Davidson et al. (2006) and de Silva et al. (2015) are essentially 2D, we do not expect significant differences in the behaviour of the BLs. An analysis of structure functions in the inertial range in the bulk of $2 \mathrm{D} R \mathrm{~B}$ convection is reported by Mazzino (2017).

A DNS of channel flow was performed using the fourth-order code described in Chung, Monty \& Ooi (2014) at $R e_{\tau}=h U_{\tau} / v=590$, where $h$ is half the channel height. The periodic (in streamwise and spanwise directions) box of size $12 h \times 4 h \times 2 h$ was discretized by $640 \times 320 \times 240$ grid points in the streamwise, spanwise and wall-normal directions, respectively. A passive scalar with $\operatorname{Pr}=1$ was added with values fixed to -0.5 at the bottom wall and 0.5 at the top wall as boundary conditions.

Convergence of the statistics computed from both datasets was checked by plotting the premultiplied probability density function of $\Delta \theta$ at various $r$, and found acceptable up to tenth order.

\section{Results}

\subsection{Direct analysis of the scaling in the energy-containing range in $R B$ convection}

We begin by plotting structure functions of second, fourth and tenth order from 2D $\mathrm{RB}$ convection as a function of $r / z$ in figure 2 . For all cases, we present results at three distances from the wall, namely $z^{+}=30$ (figure $2 a-c$ ), $z^{+}=100$ (figure $2 d-f$ ) and $z^{+}=200$ (figure $2 g-i$ ), where as usual the superscript + indicates normalization by $v / U_{\tau}$. Clearly, there is no scaling according to a scalar equivalent of (1.1) for either $R a$ at the position closest to the wall (figure $2 a-c$ ). However, in the other cases an approximately linear region appears for $r / z>1$, making it tempting to fit the slopes directly. A fitting range $5 z \leqslant r \leqslant 0.5 L$ (indicated by crosses in the figure) captures this region quite well for all $R a$ and the resulting fits are shown as red dashed lines. No fits are computed at the lowest $R a$, where the fitting range becomes prohibitively small.

A detailed comparison of the values of $D_{p}^{\theta}$ obtained in this way (figure $3 a$ ) reveals that the results depend on both $R a$ and $z^{+}$in the investigated range. Generally, values of $D_{p}^{\theta}$ are higher at $z^{+}=100$, and this difference becomes larger with increasing $R a$. In most cases, $D_{p}^{\theta}$ is very low and it is only at $z^{+}=100$ and $R a=10^{14}$ (and small $p$ ) that the values of $D_{p}^{\theta}$ are of comparable magnitude to results for $D_{p}^{u}$ in high-Re turbulent boundary layers (TBLs). We point out that the decrease of $D_{p}^{\theta}$ with increasing $p$ observed in some cases at higher $p$ is unphysical and likely related to the insufficient fitting range at higher orders (potentially in combination with somewhat inferior convergence at the highest orders). We further emphasize that even at high $R e$ a direct match between $D_{p}^{\theta}$ and $D_{p}^{u}$ is not necessarily expected. It is well established (see for example Warhaft 2000, for passive scalars) that intermittency in the inertial range $\eta \ll r \ll z$ is higher for scalars as compared to the velocity itself. This translates to lower scaling exponents $\xi_{2 p}$ in the corresponding scaling relation $S_{1}^{\theta} \sim(r / z)^{\xi_{2 p}^{\theta} / p}$ as compared to the velocity counterpart $\xi_{2 p}^{u}$. From matching the inertial scaling with (1.1) at $r=z$ de Silva et al. (2015) semiempirically derived a linear relationship between $D_{p}^{u}$ and $\xi_{2 p}^{u}$, suggesting that (disregarding other dependencies) these differences may persist 
D. Krug, X. Zhu, D. Chung, I. Marusic, R. Verzicco and D. Lohse
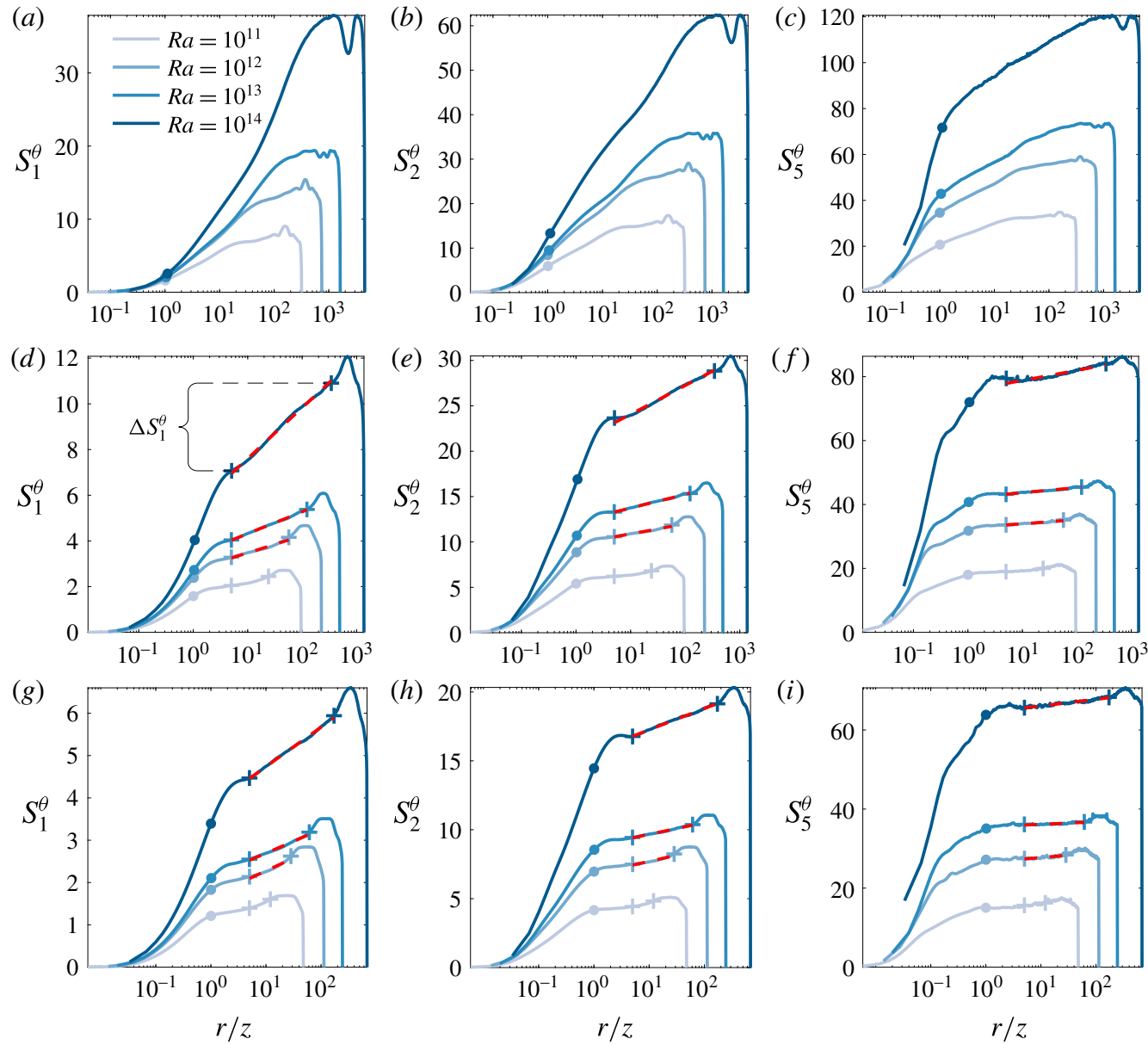

FIgURE 2. Temperature structure functions of second $(a, d, g)$, fourth $(b, e, h)$ and tenth order $(c, f, i)$ from 2D RB convection at three different locations off the wall: $z^{+}=30$ $(a-c), z^{+}=100(d-f)$ and $z^{+}=200(g-i)$ for various Rayleigh numbers. The legend in $(a)$ applies to all panels. Red dashed lines represent fits in the range $5 z \leqslant r \leqslant 0.5 L$, symbols mark $r / z=1$ (circles) and the extent of the fitting range (crosses), respectively, for later reference.

in the energy-containing range investigated here. However, a definitive answer to this question will have to be based on high-Re data of the scalar field in wall-bounded turbulent flows.

While the direct analysis of the slopes $D_{p}^{\theta}$ remains inconclusive, additional insight can be gained from studying the second-order structure function $S_{1}^{\theta}$. In this case, the difference $\Delta S_{1}^{\theta}=S_{1}^{\theta}\left(r_{2}\right)-S_{1}^{\theta}\left(r_{1}\right)$ between the structure function at two different separation distances $r_{1}$ and $r_{2}$ can be interpreted as the contribution of eddies with sizes in the range between $r_{1}$ and $r_{2}$ to the overall (scalar) energy. Consequently, when using the bounds of the log-linear scaling regime for $r_{1}, r_{2}$, as indicated in figure $2(d)$, this increment $\Delta S_{1}^{\theta}$, characterizes the contribution of the energy-containing range to the overall energy. Note that here we adopted the bounds of the fitting region used above for simplicity, but other reasonable choices give qualitatively similar results. 

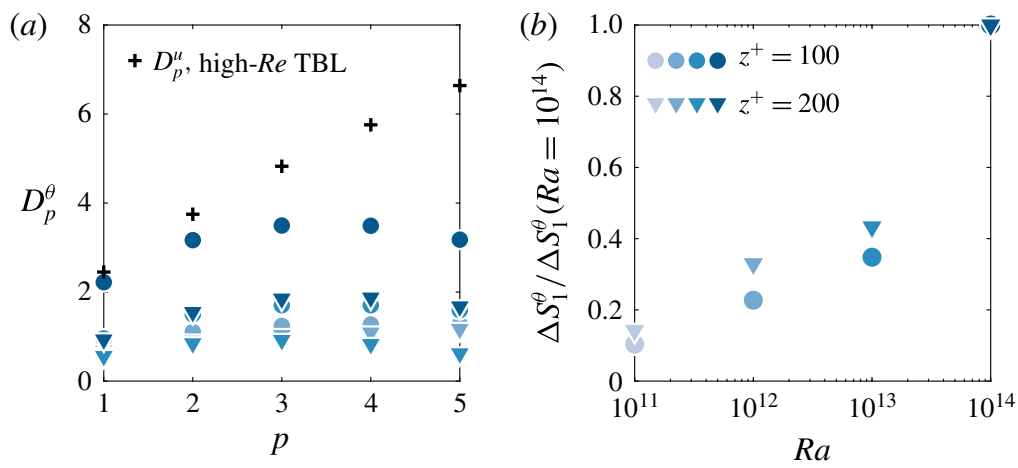

FIGURE 3. (a) Slopes $D_{p}^{\theta}$ obtained from directly fitting the structure functions in the range $5 z \leqslant r \leqslant 0.5 L$ as indicated in figure 2 compared to high- $R e_{\tau}$ reference data from de Silva et al. (2015). (b) $\Delta S_{1}^{\theta}$ as a function of $R a$ normalized by $\Delta S_{1}^{\theta}\left(R a=10^{14}\right)$. Colours indicate increasing $R a$ from light to dark (see legend in figure 2).

Figure $3(b)$, where $\Delta S_{1}^{\theta}$ is normalized by the result obtained at $R a=10^{14}$, shows that this quantity only increases mildly at low $R a$. However, it rises steeply between $R a=10^{13}$ and $R a=10^{14}$, consistent with transitional behaviour in this range. No significant differences arise between $z^{+}=100$ and $z^{+}=200$ in this case.

\subsection{Scalar ESS scaling in RB convection}

We now focus on the relative scalings $D_{p}^{\theta} / D_{1}^{\theta}$ according to (1.4). The ESS framework has been demonstrated to extend the scaling regime not only to low $R e$ but also to a wider range of wall-normal distances. In particular, Krug et al. (2017) found convincing scaling for $D_{p}^{u} / D_{1}^{u}$ as low as $z^{+}=30$. From figure $4(a-c)$, it is clear that the same does not hold for the scalar structure functions in 2D RB convection. Even at the highest $R a$, there is no linear relationship between $S_{1}^{\theta}$ and $S_{p}^{\theta}$ for any orders considered at this location. The situation improves at $z^{+}=100$ (figure $4 d-f$ ), where particularly at the highest $R a$ and low orders the curves begin to exhibit an approximately linear range. However, deviations become more apparent with increasing $p$, and results at the higher orders in figure $4(e, f)$ demonstrate that the ESS scaling is not yet fully attained at this position. It is only at $z^{+}=200$ (figure $4 g-i$ ) that a convincing ESS scaling according to (1.4) is obtained up to tenth order. The scaling range is well established at $R a=10^{14}$, already significantly decreases in size at $R a=10^{13}$, and is basically non-existent at $R a=10^{11}$. This behaviour is in very good correspondence to the changes in the $\mathrm{Nu}$ scaling in figure $1(a)$, corroborating that the change in scaling observed there is indeed due to a transition in the BL structure from laminar (no scaling) to turbulent (with ESS scaling). It should be noted that, just as for velocity structure functions, ESS scaling at $z^{+}=200$ is observed for $r / z \gtrsim 1$ for all $R a$, i.e. the spatial scaling range is the same. However, consistent with the results in figures 2 and $3(b)$, there is hardly any energy in this range at $R a$ below transition to the ultimate regime. Remarkably, the relative slopes attained for the temperature structure functions in 2D RB convection at $z^{+}=200$ appear to be the same as those measured for their velocity counterparts and reported in de Silva et al. (2017). This is remarkable since, as pointed out in $\S 3.1$, the directly measured slopes $D_{p}^{\theta}$ and $D_{p}^{u}$ need not be the same. 

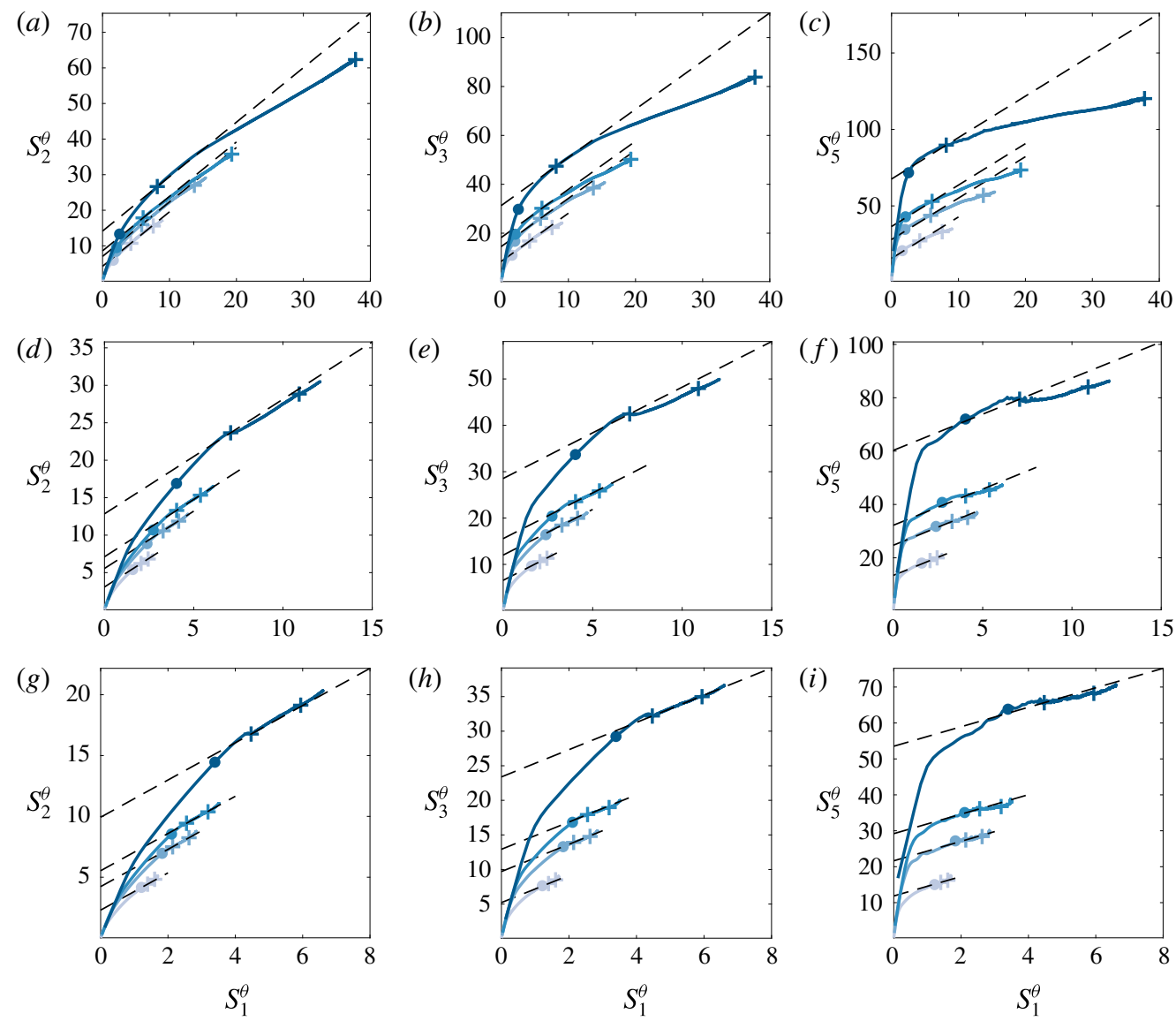

FIGURE 4. Scalar structure functions from 2D RB convection plotted versus $S_{1}$ at $z^{+}=30$ $(a-c), z^{+}=100(d-f)$, and $z^{+}=200(g-i)$ for various $R a$ (legend of figure 2 applies). Dashed lines correspond to the relative slopes $D_{p}^{u} / D_{1}^{u}$ from de Silva et al. (2017) with the $y$-axis cutoff fit at $S_{1}(r=5 z)$, symbols mark the locations of $r / z=1$ (circles) and the fitting region indicated in figure 2 (crosses) for reference.

\subsection{Scalar ESS scaling in channel flow}

The question remains why ESS scaling is only observed at a larger distance from the wall in the present case as compared to previous findings for velocity structure functions. At this point, possible explanations to be considered are that this might be either a consequence of the $2 \mathrm{D}$ set-up, a property of RB convection or a feature of the scalar field. To address this, we present ESS results of scalar structure functions in turbulent channel flow in figure $5(a-c)$ at different orders. Indeed, the results are very similar to what was observed for $2 \mathrm{D}$ RB convection before, in that there is no ESS scaling for $z^{+}=30$ and $z^{+}=100$. And again ESS scaling is recovered at $z^{+}=200$, with relative slopes matching those measured for velocity structure functions, mirroring the observations made for RB convection. So the onset of ESS scaling slightly further off the wall seems to be a general feature of scalar fields. 

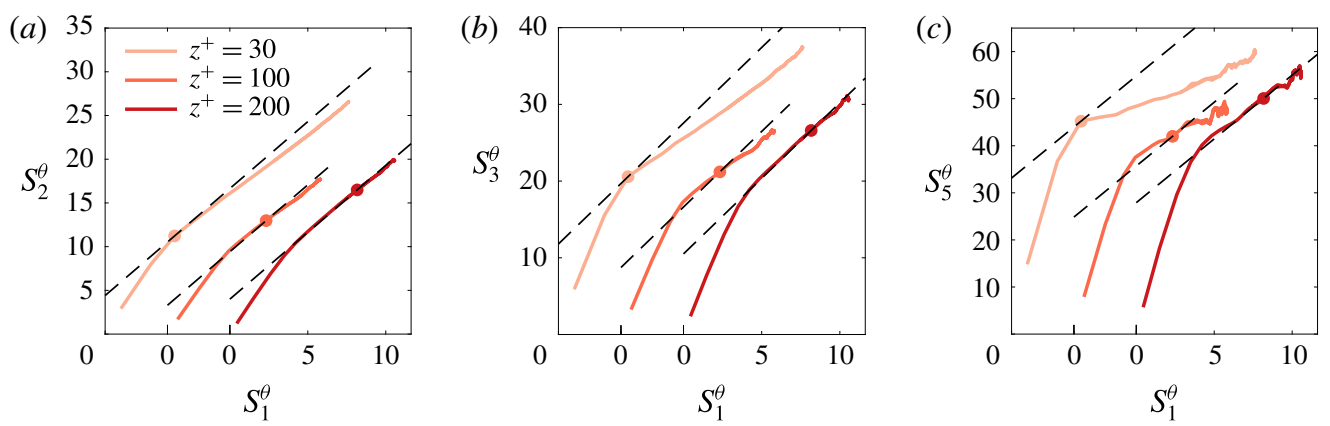

FIGURE 5. Structure function results for a passive scalar in turbulent channel flow at $R e_{\tau}=590$ in ESS form at fourth $(a)$, sixth $(b)$ and tenth order $(c)$ relative to $S_{1}^{\theta}$. Results at different $z^{+}$are shifted by 4 on the $x$-axis for clarity. Dashed lines correspond to the relative slopes $D_{p}^{u} / D_{1}^{u}$ from de Silva et al. (2017) and circles indicate the location where $r=z$ for reference.

\section{Discussion and conclusions}

We have analysed the temperature boundary layers in the energy-containing range of $2 \mathrm{D} \mathrm{RB}$ convection by means of temperature structure functions. Even though the $\mathrm{RB}$ structure functions exhibit $\log$-linear scaling for $r / z \gtrsim 1$ when plotted against separation distance, the slopes remain small at low $R a$. They were also found to vary significantly with both $R a$ and $z^{+}$, rendering the analysis inconclusive in this point. While a dependence of the slopes on $R e_{\tau}$ and wall-normal position is also observed for velocity structure functions (see Krug et al. 2017), typically the loglinear scaling is much less evident in these cases compared to figure 2 at $z^{+} \geqslant 100$. Also for the scalar in channel flow investigated here (plots not shown), a direct scaling regime is not discernible, such that it appears likely that the more prominent loglinear regimes in figure 2 are a consequence of the $2 \mathrm{D}$ set-up. An important point remains, however, that the contribution of the energy-containing range to the total energy increases significantly beyond $R a=10^{13}$, which coincides with the transition in $\mathrm{Nu}$ versus $\mathrm{Ra}$ scaling.

The main finding of the paper is the clear evidence that the temperature structure function in the BLs of turbulent 2D RB flows exhibit ESS scaling in the energycontaining range for large enough wall distances $z^{+} \gtrsim 100$. The extent of the scaling range thereby reflects the behaviour of $\mathrm{Nu}$ very well in increasing from non-existent at $R a=10^{11}$ (well in the classical regime) to considerable beyond the $N u$ scaling transition at $R a=10^{14}$. This provides further evidence that the said transition is indeed related to a switch from laminar type to turbulent type thermal BLs. Given that the underlying scaling argument is 2D (Davidson et al. 2006; de Silva et al. 2015), we would expect a similar behaviour also in a 3D flow.

Moreover, we establish that the relative slopes for scalar structure functions in the ESS form are the same as those previously obtained when analysing velocity BLs. This is confirmed by comparing the RB results to those obtained in a planar channel geometry. In both cases, ESS scaling is only established at $z^{+}=200$, which is different from velocity structure functions and appears to be a feature of the scalar field. Interestingly enough, already Perry \& Chong (1982) pointed out differences between the scalar and the velocity field. They argued that at the end of the 'lifetime' of an eddy, the vorticity contributions in the two rods of the assumed hairpin cancel 


\section{Krug, X. Zhu, D. Chung, I. Marusic, R. Verzicco and D. Lohse}

such that no induced velocity field remains. However, such a cancellation does not apply for the scalar transported by the eddy, such that the 'debris', as they called it, of past eddies sets up a scalar background profile. However, if and how exactly this is related to the observations made here remains unclear.

At least for the present cases, there also appears to be no difference between active (in RB flow) and passive (in the channel flow) scalars. In other regards, the deviations close to the wall underline, in addition to the fact the observed values of $D_{p} / D_{m}$ are sub-Gaussian, that the ESS scaling and the values of the relative slopes are indeed non-trivial.

\section{Acknowledgements}

The work was financially supported by NWO-I and the Netherlands Center for Multiscale Catalytic Energy Conversion (MCEC), both sponsored by the Netherlands Organization for Scientific Research (NWO). Part of the simulations were carried out on the Dutch national e-infrastructure with the support of SURF Cooperative. We also acknowledge PRACE for awarding us access to Marconi based in Italy at CINECA under PRACE Project No. 2016143351 and the DECI resource Archer based in the United Kingdom at Edinburgh with support from the PRACE aisbl under Project No. 13DECI0246. We also acknowledge the support of the Australian Research Council and the McKenzie Fellowship Program at the University of Melbourne.

\section{References}

Ahlers, G., Bodenschatz, E. \& He, X. 2014 Logarithmic temperature profiles of turbulent Rayleigh-Bénard convection in the classical and ultimate state for a Prandtl number of 0.8 . J. Fluid Mech. 758, 436-467.

Ahlers, G., Grossmann, S. \& Lohse, D. 2009 Heat transfer and large scale dynamics in turbulent Rayleigh-Bénard convection. Rev. Mod. Phys. 81 (2), 503-537.

Benzi, R., Ciliberto, S., Baudet, C. \& Chavarria, G. R. 1995 On the scaling of threedimensional homogeneous and isotropic turbulence. Physica D 80 (4), 385-398.

Benzi, R., Ciliberto, S., Tripiccione, R., Baudet, C., Massaioli, F. \& Succi, S. 1993 Extended self-similarity in turbulent flows. Phys. Rev. E 48 (1), R29-R32.

Chillà, F. \& Schumacher, J. 2012 New perspectives in turbulent Rayleigh-Bénard convection. Eur. Phys. J. E 35 (7), 58.

Chung, D., Monty, J. P. \& OoI, A. 2014 An idealised assessment of Townsend's outer-layer similarity hypothesis for wall turbulence. J. Fluid Mech. 742, R3.

Davidson, P. A., Nickels, T. B.\& Krogstad, P.-A. 2006 The logarithmic structure function law in wall-layer turbulence. J. Fluid Mech. 550, 51-60.

Grossmann, S. \& LohSE, D. 2000 Scaling in thermal convection: a unifying theory. J. Fluid Mech. 407, 27-56.

Grossmann, S. \& Lohse, D. 2011 Multiple scaling in the ultimate regime of thermal convection. Phys. Fluids 23 (4), 045108.

Hartmann, D. L., MoY, L. A. \& Fu, Q. 2001 Tropical convection and the energy balance at the top of the atmosphere. J. Clim. 14 (24), 4495-4511.

He, X., Funfschilling, D., Bodenschatz, E. \& Ahlers, G. $2012 a$ Heat transport by turbulent Rayleigh-Bénard convection for $\operatorname{Pr} \approx 0.8$ and $4 \times 10^{11} \lesssim R a \lesssim 2 \times 10^{14}$ : ultimate-state transition for aspect ratio $\gamma=1.00$. New J. Phys. 14 (6), 063030.

He, X., Funfschilling, D., Nobach, H., Bodenschatz, E. \& Ahlers, G. $2012 b$ Transition to the ultimate state of turbulent Rayleigh-Bénard convection. Phys. Rev. Lett. 108 (2), 024502.

He, X., van Gils, D. P. M., Bodenschatz, E. \& Ahlers, G. 2014 Logarithmic spatial variations and universal $f^{-1}$ power spectra of temperature fluctuations in turbulent Rayleigh-Bénard convection. Phys. Rev. Lett. 112 (17), 174501. 


\section{ESS scaling of temperature structure functions in $R B$ turbulence}

He, X., van Gils, D. P. M., Bodenschatz, E. \& Ahlers, G. 2015 Reynolds numbers and the elliptic approximation near the ultimate state of turbulent Rayleigh-Bénard convection. New $J$. Phys. 17 (6), 063028.

Kraichnan, R. H. 1962 Turbulent thermal convection at arbitrary Prandtl number. Phys. Fluids 5 (11), 1374-1389.

Krug, D., Yang, X. I. A., De Silva, C. M., Ostilla-Mónico, R., Verzicco, R., Marusic, I. \& LOHSE, D. 2017 Statistics of turbulence in the energy-containing range of Taylor-Couette compared to canonical wall-bounded flows. J. Fluid Mech. 830, 797-819.

Linden, P. F. 1999 The fluid mechanics of natural ventilation. Annu. Rev. Fluid Mech. 31 (1), 201-238.

LOHSE, D. \& XIA, K.-Q. 2010 Small-scale properties of turbulent Rayleigh-Bénard convection. Annu. Rev. Fluid Mech. 42, 335-364.

MALKus, W. V. R. VR 1954 The heat transport and spectrum of thermal turbulence. Proc. R. Soc. Lond. A 225 (1161), 196-212.

Mazzino, A. 2017 Two-dimensional turbulent convection. Phys. Fluids 29 (11), 111102.

Perry, A. E. \& Chong, M. S. 1982 On the mechanism of wall turbulence. J. Fluid Mech. 119, 173-217.

VAn Der Poel, E. P., Ostilla-Mónico, R., Donners, J. \& Verzicco, R. 2015 A pencil distributed finite difference code for strongly turbulent wall-bounded flows. Comput. Fluids 116, 10-16.

van Der Poel, E. P., Stevens, R. J. A. M. \& Lohse, D. 2013 Comparison between two-and three-dimensional Rayleigh-Bénard convection. J. Fluid Mech. 736, 177-194.

RAHMSTORF, S. 2000 The thermohaline ocean circulation: a system with dangerous thresholds? Clim. Change 46 (3), 247-256.

Schlichting, H. \& Gersten, K. 2000 Boundary-layer Theory, 8th edn. Springer.

Schmalzl, J., BReuer, M. \& HAnsen, U. 2004 On the validity of two-dimensional numerical approaches to time-dependent thermal convection. Europhys. Lett. 67 (3), 390-396.

De Silva, C. M., Krug, D., Lohse, D. \& Marusic, I. 2017 Universality of the energy-containing structures in wall-bounded turbulence. J. Fluid Mech. 823, 498-510.

de Silva, C. M., Marusic, I., Woodcock, J. D. \& Meneveau, C. 2015 Scaling of second-and higher-order structure functions in turbulent boundary layers. J. Fluid Mech. 769, 654-686.

Spiegel, E. A. 1971 Convection in stars I. Basic Boussinesq convection. Annu. Rev. Astron. Astrophys. 9 (1), 323-352.

Townsend, A. A. 1976 The Structure of Turbulent Shear Flow. Cambridge University Press.

Warhaft, Z. 2000 Passive scalars in turbulent flows. Annu. Rev. Fluid Mech. 32 (1), 203-240.

Zhu, X., Mathai, V., Stevens, R. J. A. M., Verzicco, R. \& Lohse, D. 2018 Transition to the ultimate regime in two-dimensional Rayleigh-Bénard convection. Phys. Rev. Lett. 120, 144502. 